\title{
Dominique Maingueneau (éd), « Marges linguistiques », № 9
}

\section{Rachele Raus}

\section{(2) OpenEdition}

1 Journals

\section{Édition électronique}

URL : http://journals.openedition.org/studifrancesi/30993

DOI : 10.4000/studifrancesi.30993

ISSN : 2421-5856

Éditeur

Rosenberg \& Sellier

\section{Édition imprimée}

Date de publication : 1 avril 2006

Pagination : 213-214

ISSN : 0039-2944

\section{Référence électronique}

Rachele Raus, « Dominique Maingueneau (éd), « Marges linguistiques », No 9 », Studi Francesi [En ligne], 148 (XLX | I) | 2006, mis en ligne le 30 novembre 2015, consulté le 18 avril 2021. URL : http:// journals.openedition.org/studifrancesi/30993; DOI : https://doi.org/10.4000/studifrancesi.30993

Ce document a été généré automatiquement le 18 avril 2021.

\section{(c) (i) (9)}

Studi Francesi è distribuita con Licenza Creative Commons Attribuzione - Non commerciale - Non opere derivate 4.0 Internazionale. 


\title{
Dominique Maingueneau (éd), « Marges linguistiques », № 9
}

\author{
Rachele Raus
}

\section{RÉFÉRENCE}

DOMINIQUE MAINGUENEAU (éd), « Marges linguistiques », № 9, 2005, pp. 275.

1 Ce numéro de la revue, édité par Dominique Maingueneau, fait un état de l'art de l'Analyse du discours (désormais AD) en France. On peut retracer la présence d'au moins trois pistes de recherche : la première est une réflexion sur les outils théoriques de cette "discipline carrefour"; la deuxième consiste dans l'intérêt de plus en plus marqué pour les corpus oraux, d'où le fait que les analyses soient de plus en plus redevables de l'approche interactionnelle; la troisième est l'évidence d'un ralliement de l' AD avec les disciplines sociales, l'AD consistant au fond dans l'imbrication des modalités d'énonciations et des lieux sociaux de production des discours.

Pour ce qui est du premier axe, MAINGUENEAU (L'analyse du discours et ses frontières, pp. 64-75) souligne que l'AD rassemble des chercheurs qui partagent des ressources communes dans le sens d'air de famille (cf. L. Wittgenstein). Il y a un autre paramètre à considérer pour discerner les courants différents, à savoir la territorialité des recherches. Enfin, il retrace les unités fondamentales (topiques, non - topiques) qui font l'intérêt des chercheurs. JACQUES GUILHAUMOU (Où va l'analyse de discours? Autour de la notion de formation discursive, pp. 95-114) ne partage pas l'avis de Mainguenau pour ce qui est des appareils théoriques de l'AD. Guilhaumou donne l'exemple du Dictionnaire $d$ 'AD de Maingueneau et Charaudeau (2002) pour dénoncer une approche statique aux catégories conceptuelles de l'AD ainsi qu'un éloignement d'une réflexion herméneutique qui tienne compte de la matérialité de la langue. Par contre, le dictionnaire d'AD de Detrié, Siblot et Verine (Termes et concepts pour l'analyse du discours, 2001) présenterait une approche dynamique et praxematique. Guilhaumou donne aussi l'exemple de la notion de formation discursive, en en montrant l'évolution 
conceptuelle et opérationnelle depuis les années 1970, par rapport justement aux changements épistémologiques de l'AD. AlAin RABATEL ( $a$ a part de l'énonciateur dans la construction interactionnelle des points de vue, pp. 115-36) revient sur la disjonction entre énonciateur et locuteur de Benveniste et surtout de Ducrot. Il fonde la possibilité d'une subjectivité considérée dans son actualisation déictique (locuteur) et modale (énonciateur). De même il pose la possibilité d'un proto-énonciateur, qui englobe une multiplicité de points de vue, ainsi que d'une hétérogénéité constitutive de l'énonciation. JACQUES BRES et ALEKSANDRA NOWAKOWSKA (Dis-moi avec qui tu «dialogues», je te dirai qui tu es..., pp. 137-53) reviennent sur la notion de dialogisme de Bakhtine, d'un point de vue syntactique. Ils posent l'existence d'un dialogisme interdiscursif, interlocutif et d'un autodialogisme. A partir du clivage entre un dit enchâssant et un ou plusieurs dit/s enchâssé/s, ils retracent le positionnement de l'Enonciateur. Ils posent aussi la spécificité d'un dialogisme citatif et d'un dialogisme responsif. LAURENCE ROSIER (L'analyse de discours et ses corpus à travers le prisme du discours rapporté, pp. 154-64) souligne l'intérêt de plus en plus croissant de l'AD pour les corpus oraux de même que le besoin d'une réflexion sur les outils théoriques en fonction de ces nouveaux corpus. Tout particulièrement, la notion de discours rapporté a permis à l'AD « la mise en avant de la réflexivité discursive » (pp. 162). DIANE VINCENT (Analyse conversationnelle, analyse du discours et interprétation des discours sociaux : le cas de la trash radio, pp. 165-75) analyse un corpus oral pour lier l'étude des genres aux contextes de production du discours. Elle pose aussi comme essentielle une approche pragmatique et interactionnelle, pour démontrer le procédé argumentatif et aussi manipulatif des programmes de la trash radio au Canada, où les insultes sont aussi des actes linguistiques qui agissent sur la face de l'autre.

3 ALICE TOMA (L'organisation informationnelle du discours de vulgarisation scientifique, pp. 176-194) représente de manière hiérarchisée la structuration informationnelle dans l'écrit de vulgarisation, en utilisant la méthode de l'Ecole de Genève. Cette hiérarchie fait ressortir la négociation entre l'interviewer et l'interviewé dans la co-construction de l'objet du discours. VIRGINIE ANDRÉ (Oui non : une pratique discursive sous influence, pp. 195-213) montre le rôle non seulement sémantique mais surtout pragmatique (d'atténuation et de négociation) ou argumentatif du couple « oui non » dans le genre réunion de travail «à la française ». Les facteurs extralinguistiques et l'importance du lieu de production de ce corpus oral sont également tenus en compte. VÉRONIQUE MAGAUD (Modes d'appropriation topiques et structuration d'un genre: la consultation psychologique radiophonique, pp. 214-230) revient sur la nécessité posée par les corpus oraux d'utiliser des approches et des notions étroitement liées aux genres analysés. Ainsi, le discours psychologique à la radio produit une perte de la dimension personnelle de l'analyse psychologique, pour passer à une dimension doxale par la médiation de l'animatrice du débat, et surtout de l'expert. CLARA UBALDINA IORDA MUR (La désignation dans un discours de justification: Jacques Chirac et le conflit de l'Irak, pp. 232-241) revient sur un corpus écrit pour montrer, à travers l'analyse des désignants («nous", "on», "la France»), le positionnement de Chirac vis-à-vis des journalistes et de L'onU, à l'occasion du dernier conflit en Irak. Elle s'attarde aussi sur une étude de la réception de ce même discours. Yana Grinshpun (O et Oh: une graphie entre langue et genres de discours, pp. 242-255) analyse l'oscillation graphique de l'interjection $O$ / Oh depuis le XVIe siècle. Elle y voit des raisons linguistiques mais surtout des choix liés au genre, la graphie $O$ l'emportant dans des genres ressentis comme littéraires. 